\title{
Postacceleration effects in the Coulomb dissociation of neutron halo nuclei
}

\author{
Prabir Banerjee, ${ }^{1}$ Gerhard Baur, ${ }^{2}$ Kai Hencken, ${ }^{3}$ Radhey Shyam, ${ }^{4}$ and Dirk Trautmann ${ }^{3}$ \\ ${ }^{1}$ Physics Group, Variable Energy Cyclotron Centre, Calcutta 700 064, India \\ ${ }^{2}$ Institut für Kernphysik (Theorie), Forschungszentrum Jülich, D-52425 Jülich, Germany \\ ${ }^{3}$ Institut für Physik, Universität Basel, Klingelbergstr. 82, 4056 Basel, Switzerland \\ ${ }^{4}$ Saha Institute of Nuclear Physics, Calcutta 700 064, India
}

(Received 24 January 2002; published 22 May 2002)

\begin{abstract}
We study the breakup of one-neutron halo nuclei in the Coulomb field of a target nucleus. In the post-form distorted wave Born approximation theory of this reaction, with only Coulomb distortions in the entrance and outgoing channels, an analytic solution for the breakup $T$ matrix is known. We study this $T$ matrix and the corresponding cross sections numerically. This formula can be related to the first order semiclassical treatment of the electromagnetic dissociation. This theory contains the electromagnetic interaction between the core and the target nucleus to all orders. We show that higher order effects (including postacceleration) are small in the case of higher beam energies and forward scattering. We investigate the beam energy dependence of the postacceleration effects. They are found to be quite important for smaller beam energies (slow collisions), but almost negligible at larger ones.
\end{abstract}

DOI: 10.1103/PhysRevC.65.064602

PACS number(s): 25.60.Gc, 24.10.Eq, 24.50.+g, 25.70.De

\section{INTRODUCTION}

Breakup processes, in nucleus-nucleus collisions, are complicated, in whatever way they are studied. Coulomb breakup (CB) is a significant reaction channel in the scattering of halo nuclei from a heavy target nucleus (see, e.g., [1-4]). With the operation of exotic beam facilities all over the world, these reactions (previously restricted essentially to deuteron induced reactions) have come into sharp focus again. CB provides a convenient way to put constraints on the structure of these nuclei $[5,6]$. This is of interest also for nuclear astrophysics, since the breakup cross section can be related to the photodissociation cross section and to radiative capture reactions relevant for nuclear astrophysics [7].

The breakup reactions of the halo nuclei have been investigated theoretically by several authors, using a number of different approaches (see, e.g., $[3,8]$ for an extensive list of references). One often used method has been to treat the breakup reaction as the inelastic excitation of the projectile from its ground state to the continuum [9]. The corresponding $T$ matrix is written in terms of the prior-form distorted wave Born approximation (DWBA) [10]. For pure Coulomb breakup, the semiclassical approximation of this theory is the first order perturbative Alder-Winther theory of Coulomb excitation [11]. It has recently been used to analyze the data on the breakup reactions induced by the one-neutron halo nuclei ${ }^{11} \mathrm{Be}[12]$ and ${ }^{19} \mathrm{C}$ [13]. The question of the higher order electromagnetic effects [14-18] was studied [19] recently within this framework. They were found to be small for both zero range and finite range wave functions of the relative motion of the fragments within the projectile. Also, in a dynamical description of the breakup of these nuclei where the time evolution of the projectile in coordinate space is described by solving the three-dimensional time dependent Schrödinger equation treating the projectile-target interaction as a time dependent external perturbation, the higher order effects turned out [20] to be only of the order of $10 \%$ for beam energies in the range of $60-80 \mathrm{MeV} /$ nucleon.
A direct breakup model (DBM) (which reduces to the Serber model in a particular limit [3]) has been formulated within the framework of the post-form DWBA $[8,10]$. An important advantage of this model is that it can be solved analytically for the case of the breakup of the neutron halo nuclei with the entrance and outgoing channels involving only the Coulomb distortions $[8,21,22]$. It constitutes an ideal "theoretical laboratory" to investigate the physics of the breakup reactions, its certain limiting cases, and its relation to other models like the semiclassical approximation. Particularly, the effect of postacceleration (to be explained in more detail below) can be studied in a unique way within this approach.

The aim of this paper is to investigate the role of the higher order effects (which includes postacceleration) in the Coulomb breakup of the one-neutron halo nuclei ${ }^{11} \mathrm{Be}$ and ${ }^{19} \mathrm{C}$ within the post-form DWBA theory of the breakup reactions. We calculate the triple and double differential cross sections of the fragments, observed in the breakup of these nuclei on a ${ }^{208} \mathrm{~Pb}$ target, within the exact theory as well as within its first order approximation. We also calculate the relative energy distributions of the outgoing fragments emitted in these reactions, within the two theories. Calculations have been performed for a range of beam energies in order to investigate the beam energy dependence of the higher order effects. In Sec. II, we present our theoretical model. The results of our calculations and their discussions are given in Sec. III. A summary, conclusions, and the outlook of our work in given in Sec. IV.

\section{DESCRIPTION OF THE THEORETICAL MODEL}

\section{A. Coulomb wave Born approximation}

We consider the reaction $a+t \rightarrow c+n+t$, where the projectile $a$ breaks up into the charged fragment $c$ and the neutron $n$ in the Coulomb field of a target $t$. 
The starting point of the Coulomb wave Born approximation (CWBA) is the post-form $T$ matrix of the breakup reaction which is given by

$$
\begin{aligned}
T= & \int d \xi d \mathbf{r}_{1} d \mathbf{r}_{i} \chi_{c}^{(-) *}\left(\mathbf{k}_{c}, \mathbf{r}\right) \Phi_{c}^{*}\left(\xi_{c}\right) \chi_{n}^{(-) *}\left(\mathbf{k}_{n}, \mathbf{r}_{n}\right) \\
& \times \Phi_{n}^{*}\left(\xi_{n}\right) V_{c n}\left(\mathbf{r}_{1}\right) \Psi_{a}^{(+)}\left(\xi_{a}, \mathbf{r}_{1}, \mathbf{r}_{i}\right) .
\end{aligned}
$$

The functions $\chi_{c}^{(-) *}\left(\mathbf{k}_{c}, \mathbf{r}\right)$, and $\chi_{n}^{(-) *}\left(\mathbf{k}_{n}, \mathbf{r}_{n}\right)$ are the distorted waves for the relative motions of $c$ and $n$ with respect to $t$ and the center of mass (c.m.) of the $c+t$ system, respectively. The arguments of these functions contain the corresponding Jacobi momenta and coordinates. $\Phi$ 's are the internal state wave functions of the concerned particles which depend on the internal coordinates $\xi$. The function $\Psi_{a}^{(+)}\left(\xi_{a}, \mathbf{r}_{1}, \mathbf{r}_{i}\right)$ is the exact three-body scattering wave function of the projectile with a wave vector $\mathbf{k}_{a}$, which satisfies outgoing wave boundary conditions. The vectors $\mathbf{k}_{c}$ and $\mathbf{k}_{n}$ are the Jacobi wave vectors of $c$ and $n$, respectively, in the final channel of the reaction. The function $V_{c n}\left(\mathbf{r}_{1}\right)$ represents the interaction between $c$ and $n$. For the pure Coulomb breakup case, the function $\chi_{c}^{(-)}\left(\mathbf{k}_{c}, \mathbf{r}\right)$ is taken as the Coulomb distorted wave (for a point Coulomb interaction between the charged core $\mathrm{c}$ and the target) satisfying incoming wave boundary conditions, and the function $\chi_{n}^{(-)}\left(\mathbf{k}_{n}, \mathbf{r}_{n}\right)$ is just a plane wave as there is no Coulomb interaction between the target and the neutron. The position vectors satisfy the following relations:

$$
\begin{gathered}
\mathbf{r}=\mathbf{r}_{i}-\alpha \mathbf{r}_{1}, \quad \alpha=\frac{m_{n}}{m_{a}}, \\
\mathbf{r}_{n}=\gamma \mathbf{r}_{1}+\delta \mathbf{r}_{i}, \quad \delta=\frac{m_{t}}{m_{c}+m_{t}}, \quad \gamma=(1-\alpha \delta) .
\end{gathered}
$$

In the distorted wave Born approximation, we write

$$
\Psi_{a}^{(+)}\left(\xi_{a}, \mathbf{r}_{1}, \mathbf{r}_{i}\right) \approx \Phi_{a}\left(\xi_{a}, \mathbf{r}_{1}\right) \chi_{a}^{(+)}\left(\mathbf{k}_{a}, \mathbf{r}_{i}\right)
$$

In Eq. (4), the dependence of $\Phi_{a}$ on $\mathbf{r}_{1}$ describes the relative motion of the fragments $c$ and $n$ in the ground state of the projectile. The function $\chi_{a}^{(+)}\left(\mathbf{k}_{a}, \mathbf{r}_{i}\right)$ is the Coulomb distorted scattering wave describing the relative motion of the c.m. of the projectile with respect to the target, satisfying outgoing wave boundary conditions.

The integration over the internal coordinates $\xi$, in Eq. (1), gives

$$
\begin{aligned}
& \int d \xi \Phi_{c}^{*}\left(\xi_{c}\right) \Phi_{n}^{*}\left(\xi_{n}\right) \Phi_{a}\left(\xi_{a}, \mathbf{r}_{1}\right) \\
& =\sum_{l m j \mu}\left\langle l m j_{n} \mu_{n} \mid j \mu\right\rangle\left\langle j_{c} \mu_{c} j \mu \mid j_{a} \mu_{a}\right\rangle i^{l} \Phi_{a}\left(\mathbf{r}_{1}\right),
\end{aligned}
$$

with

$$
\Phi_{a}\left(\mathbf{r}_{1}\right)=u_{l}\left(r_{1}\right) Y_{l m}\left(\hat{\mathbf{r}}_{1}\right)
$$

In Eq. (6), $l$ (the orbital angular momentum for the relative motion between fragments $c$ and $n$ ) is coupled to the spin of $n$ and the resultant channel spin $j$ is coupled to spin $j_{c}$ of the core $c$ to yield the spin of $a\left(j_{a}\right)$. The $T$ matrix can now be written as

$$
\begin{aligned}
T^{\mathrm{CWBA}}= & \sum_{l m j \mu}\left\langle l m j_{n} \mu_{n} \mid j \mu\right\rangle\left\langle j_{c} \mu_{c} j \mu \mid j_{a} \mu_{a}\right\rangle \\
& \times i^{l} \hat{l} \beta_{l m}^{\mathrm{CWBA}}\left(\mathbf{k}_{c}, \mathbf{k}_{n} ; \mathbf{k}_{a}\right),
\end{aligned}
$$

where

$$
\begin{aligned}
\hat{l} \beta_{l m}^{\mathrm{CWBA}}\left(\mathbf{k}_{c}, \mathbf{k}_{n} ; \mathbf{k}_{a}\right)= & \int d \mathbf{r}_{1} d \mathbf{r}_{i} \chi_{c}^{(-) *}\left(\mathbf{k}_{c}, \mathbf{r}\right) e^{-i \mathbf{k}_{n} \cdot \mathbf{r}_{n} V_{c n}\left(\mathbf{r}_{1}\right)} \\
& \times u_{l}\left(r_{1}\right) Y_{l m}\left(\hat{r}_{1}\right) \chi_{a}^{(+)}\left(\mathbf{k}_{a}, \mathbf{r}_{i}\right)
\end{aligned}
$$

with $\hat{l} \equiv \sqrt{2 l+1}$.

Equation (8) involves a six-dimensional integral which makes its computation quite complicated. The problem gets further acute because the integrand has a product of three scattering waves that exhibit an oscillatory behavior asymptotically. Therefore, approximate methods have been used, such as the zero-range approximation (ZRA) in which we write $V_{c n}\left(\mathbf{r}_{1}\right) \Phi_{a}\left(\mathbf{r}_{1}\right)=D_{0} \delta\left(\mathbf{r}_{1}\right)$, with $D_{0}$ being the zerorange normalization constant (see, e.g., [23-25]), or the approximation used in [21], where the projectile c.m. coordinate is replaced by that of the core-target system (i.e., $\mathbf{r}_{i}$ $\approx \mathbf{r})$. Both these methods lead to a factorization of the amplitude [Eq. (8)] into two independent parts, which reduces the computational complexity to a great extent. However, the application of both these methods to the reactions of halo nuclei is questionable (see, e.g., [8], for a detailed discussion).

In the finite-range CWBA theory [8], the Coulomb distorted wave of particle $c$, in the final channel, is written as

$$
\chi_{c}^{(-)}\left(\mathbf{k}_{c}, \mathbf{r}\right)=e^{-i \alpha \mathbf{K} \cdot \mathbf{r}_{1}} \chi_{c}^{(-)}\left(\mathbf{k}_{c}, \mathbf{r}_{i}\right) .
$$

Equation (9) represents an exact Taylor series expansion about $\mathbf{r}_{i}$ if $\mathbf{K}=-i \nabla_{\mathbf{r}_{i}}$ is treated exactly. However, instead of doing this we employ a local momentum approximation $[26,27]$, where the magnitude of momentum $\mathbf{K}$ is taken to be

$$
K(R)=\sqrt{\frac{2 m}{\hbar^{2}}[E-V(R)] .}
$$

Here $m$ is the reduced mass of the $c$ - $t$ system, $E$ is the energy of particle $c$ relative to the target in the c.m. system, and $V(R)$ is the Coulomb potential between $c$ and the target $t$ separated by the distance $R$. Thus, the magnitude of the momentum $\mathbf{K}$ is evaluated at some separation $R$ which is held fixed for all the values of $r$. The value of $R$ is taken to be 10 $\mathrm{fm}$, which is the same as that used in [8]. For further details and a discussion of the validity of this approximation, we refer to $[8,28]$.

On substituting Eq. (9) into Eq. (8), we obtain the following factorized form of the amplitude $\beta_{l m}^{\mathrm{CWBA}}$ : 


$$
\begin{aligned}
& \hat{l} \beta_{l m}^{\mathrm{CWBA}}\left(\mathbf{k}_{c}, \mathbf{k}_{n} ; \mathbf{k}_{a}\right) \\
& \quad=Z_{l m} \int d \mathbf{r}_{i} \chi_{c}^{(-)} *\left(\mathbf{k}_{c}, \mathbf{r}_{i}\right) e^{-i \delta \mathbf{k}_{n} \cdot \mathbf{r}_{i}} \chi_{a}^{(+)}\left(\mathbf{k}_{a}, \mathbf{r}_{i}\right),
\end{aligned}
$$

where

$$
Z_{l m}=\int d \mathbf{r}_{1} e^{-i \mathbf{k}_{1} \cdot \mathbf{r}_{1}} V_{c n}\left(\mathbf{r}_{1}\right) u_{l}\left(r_{1}\right) Y_{l m}\left(\hat{r}_{1}\right),
$$

where $\mathbf{k}_{1}=\gamma \mathbf{k}_{n}-\alpha \mathbf{K}$.

This amplitude differs from that of the ZRA studied earlier [10] as it allows the use of the full wave function for the relative motion of the fragments (corresponding to any value of $l$ ) in the ground state of the projectile. However, it should be stressed that as far as the postacceleration effects are concerned, both amplitudes would lead to identical results.

The triple-differential cross section of the reaction is given by

$$
\frac{d^{3} \sigma}{d E_{c} d \Omega_{c} d \Omega_{n}}=\frac{2 \pi}{\hbar v_{a}} \rho\left(E_{c}, \Omega_{c}, \Omega_{n}\right) \sum_{l m}\left|\beta_{l m}^{\mathrm{CWBA}}\right|^{2},
$$

where $\rho\left(E_{c}, \Omega_{c}, \Omega_{n}\right)$ is the appropriate three-body phase space factor [8] and $v_{a}$ the velocity of particle $a$.

On substituting the Coulomb distorted waves

$$
\begin{aligned}
& \chi_{c}^{(-) *}\left(\mathbf{k}_{c}, \mathbf{r}_{i}\right)=e^{-\pi \eta_{c} / 2} \Gamma\left(1+i \eta_{c}\right) \\
& \times e^{-i \mathbf{k}_{c} \cdot \mathbf{r}_{i} F_{1}} F_{1}\left(-i \eta_{c}, 1, i\left(k_{c} r_{i}+\mathbf{k}_{c} \cdot \mathbf{r}_{i}\right)\right), \\
& \chi_{a}^{(+)}\left(\mathbf{k}_{a}, \mathbf{r}_{i}\right)=e^{-\pi \eta_{a} / 2} \Gamma\left(1+i \eta_{a}\right) \\
& \times e^{i \mathbf{k}_{a} \cdot \mathbf{r}_{i}} F_{1}\left(-i \eta_{a}, 1, i\left(k_{a} r_{i}-\mathbf{k}_{a} \cdot \mathbf{r}_{i}\right)\right)
\end{aligned}
$$

into Eqs. (11) and (13), one gets, for the triple-differential cross section,

$$
\begin{aligned}
\frac{d^{3} \sigma}{d E_{c} d \Omega_{c} d \Omega_{n}}= & \frac{32 \pi^{4}}{\hbar v_{a}} \rho\left(E_{c}, \Omega_{c}, \Omega_{n}\right) \\
& \times \frac{\eta_{a} \eta_{c}}{\left(e^{2 \pi \eta_{c}}-1\right)\left(e^{\left.2 \pi \eta_{a}-1\right)}\right.}|I|^{2} \sum_{l}\left|Z_{l}^{\prime}\right|^{2} .
\end{aligned}
$$

In Eqs. (14)-(16), $\eta$ 's are the Coulomb parameters for the respective particles. In Eq. (16), $I$ is the Bremsstrahlung integral [29] which can be evaluated in the closed form

$$
\begin{aligned}
I= & -i\left[B ( 0 ) ( \frac { d D } { d \Lambda } ) _ { \Lambda = 0 } ( - \eta _ { a } \eta _ { c } ) _ { 2 } F _ { 1 } \left(1-i \eta_{a}, 1\right.\right. \\
& \left.\left.-i \eta_{c} ; 2 ; D(0)\right)+\left(\frac{d B}{d \Lambda}\right)_{\Lambda=02} F_{1}\left(-i \eta_{a},-i \eta_{c} ; 1 ; D(0)\right)\right],
\end{aligned}
$$

where

$$
\begin{aligned}
B(\Lambda)= & \frac{4 \pi}{k^{2\left(i \eta_{a}+i \eta_{c}+1\right)}}\left[( k ^ { 2 } - 2 \mathbf { k } \cdot \mathbf { k } _ { a } - 2 \Lambda k _ { a } ) ^ { i \eta _ { a } } \left(k^{2}-2 \mathbf{k} \cdot \mathbf{k}_{c}\right.\right. \\
& \left.\left.-2 \Lambda k_{c}\right)^{i \eta_{c}}\right], \\
D(\Lambda)= & \frac{2 k^{2}\left(k_{a} k_{c}+\mathbf{k}_{a} \cdot \mathbf{k}_{c}\right)-4\left(\mathbf{k} \cdot \mathbf{k}_{a}+\Lambda k_{a}\right)\left(\mathbf{k} \cdot \mathbf{k}_{c}+\Lambda k_{c}\right)}{\left(k^{2}-2 \mathbf{k} \cdot \mathbf{k}_{a}-2 \Lambda k_{a}\right)\left(k^{2}-2 \mathbf{k} \cdot \mathbf{k}_{c}-2 \Lambda k_{c}\right)},
\end{aligned}
$$

with

$$
\mathbf{k}=\mathbf{k}_{a}-\mathbf{k}_{c}-\delta \mathbf{k}_{n} .
$$

The factor $Z_{l}^{\prime}$ contains the projectile structure information and is given by

$$
Z_{l}^{\prime}=\int d r_{1} r_{1}^{2} j_{l}\left(k_{1} r_{1}\right) V_{c n}\left(r_{1}\right) u_{l}\left(r_{1}\right) .
$$

It may be noted that the triple-differential cross sections with respect to relative and c.m. coordinates of the fragments are related to those given by Eq. (13) as

$$
\frac{d^{3} \sigma}{d E_{c-n} d \Omega_{c-n} d \Omega_{t-(c+n)}}=J\left[\frac{d^{3} \sigma}{d E_{c} d \Omega_{c} d \Omega_{n}}\right],
$$

where the form of the Jacobian $J$ is the same as that given in [30]. In Eq. (22), $t-(c+n)$ corresponds to the coordinates of the relative motion of the c.m. of the fragments $c$ and $n$ with respect to the target, while $c-n$ corresponds to that of the relative motion between them.

The CWBA description [Eqs. (11) and (12)] includes the effects of postacceleration, which refers to the situation where the core $c$ has a larger final state energy than what one gets from sharing the kinetic energy among the fragments according to their mass ratio. This effect arises in a purely classical picture [31] of the breakup process. The nucleus $a$ $=(c+n)$ moves up the Coulomb potential, losing the appropriate amount of kinetic energy. At an assumed "breakup point," this kinetic energy (minus the binding energy) is supposed to be shared among the fragments according to their mass ratio (assuming that the velocities of $c$ and $n$ are equal). Running down the Coulomb barrier, the charged particle $c$ alone (and not the neutron) gains back the Coulomb energy, resulting in its postacceleration. Of course this picture is based on the purely classical interpretation of this process, and will be modified in a quantal treatment, where such a "breakup point" does not exist. Postacceleration is clearly observed in the low energy deuteron breakup, both in the theoretical calculations and in the corresponding experiments (see, e.g., $[10,32])$. However, in the description of the Coulomb dissociation of halo nuclei at high beam energies within this theory $[8,22,33]$, the postacceleration effects become negligibly small. We shall investigate this point further for the ${ }^{11} \mathrm{Be}$ and ${ }^{19} \mathrm{C}$ Coulomb dissociation experiments $[12,13]$. On the other hand, in semiclassical Coulomb excitation theory the higher order effects have been found [19] to be small, for both zero-range as well as finite-range wave functions of the $c+n$ system. 
One can establish a relation between the apparently very different CWBA and the semiclassical theory. It was recently noticed [35] that in the limit of the Coulomb parameter, $\eta_{a}$ $\ll 1$ (i.e., in the Born approximation), both theories give the same result. It was further found that this agreement is also valid for arbitrary values of $\eta_{a}$ and $\eta_{c}$, provided the beam energies are high as compared to the relative energy $\left(E_{c n}\right)$ of fragments $c$ and $n$ in the ground state of the projectile. The first order approximation to the amplitude given by Eq. (11) can be written as [35]

$$
\begin{aligned}
\hat{l} \beta_{l m}^{\text {first order }}= & 4 \pi Z_{l m} f_{\mathrm{Coul}} e^{-\pi \xi / 2}\left[e^{-i \phi} \frac{1}{k_{a}^{2}-\left[\boldsymbol{k}_{c}+\delta \boldsymbol{k}_{n}\right]^{2}}\right. \\
& \left.+e^{i \phi} \frac{m_{c}}{m_{a}} \frac{1}{\left[k_{c}^{2}-\left(\delta \boldsymbol{k}_{n}-\boldsymbol{k}_{a}\right)^{2}\right]}\right],
\end{aligned}
$$

where the relative phase $\phi=\sigma\left(\eta_{c}\right)-\sigma\left(\eta_{a}\right)-\sigma(\xi)$ $-\xi /[2 \ln |D(0)|]$, with $\sigma(\eta)$ being the usual Coulomb phase shifts and $\xi=\eta_{c}-\eta_{a}$. In Eq. (23), we have defined $f_{\text {Coul }}$ $=2 \eta_{a} k_{a} / k^{2}$. This term is very similar to the Born approximation (BA) result given in [34]; in the limit $\xi \rightarrow 0$ it actually coincides with the BA expression. This equation can be used to investigate the role of higher order effects. It may be noted that the derivation of Eq. (23) makes use only of the condition that $-D(0) \gg 1$ which is met for beam energies large as compared to the binding energy. A further approximate form of this equation can be obtained for small momentum transfer $k$ which is discussed below.

\section{B. Scaling properties}

In many experimental situations, the momentum transfer $\boldsymbol{k}$ [Eq. (20)] is small. In that case, one can expand Eq. (23) (with $\phi=\xi=0$ ) for small values of $k$ to obtain an approximate first order amplitude

$$
\hat{l} \overline{\boldsymbol{\beta}}_{l m}^{\text {first order }}=f_{\text {Coul }} \frac{2 Z_{l m}}{\pi^{2}} \frac{m_{n}^{2} m_{c}}{\left(m_{n}+m_{c}\right)^{3}} \frac{2 \boldsymbol{q} \cdot \boldsymbol{k}}{\left(\kappa^{2}+q^{2}\right)^{2}},
$$

where the relative momentum between $c$ and $n$ is given by $\boldsymbol{q}=\left(m_{c} \boldsymbol{k}_{n}-m_{n} \boldsymbol{k}_{c}\right) /\left(m_{n}+m_{c}\right)$ and $\kappa$ is related to the $c-n$ separation energy in the ground state of the projectile, $E_{c n}^{\text {bind }}\left(=\hbar^{2} \kappa^{2} / 2 \mu\right.$, with $\mu$ being the reduced mass of the $c-n$ system). This result is in remarkable agreement with the usual first order treatment of the electromagnetic excitation in the semiclassical approximation.

In the semiclassical approach, the scattering amplitude is given by the elastic scattering (Rutherford) amplitude times an excitation amplitude $a(b)$, where the impact parameter $b$ is related to the transverse momentum transfer $q_{\perp}$ and $\eta_{a}$ by $b=2 \eta_{a} \hbar / q_{\perp}$. The absolute square of $a(b)$ gives the breakup probability $P(b)$, which, in the lowest order (LO), is given by $[34,19]$

$$
\frac{d P_{\mathrm{LO}}}{d q}=\frac{16 y^{2}}{3 \pi \kappa} \frac{x^{4}}{\left(1+x^{2}\right)^{4}},
$$

where the variable $x$ is related to the relative momentum between $n$ and $c$ by $x=q / \kappa$ and $y$ is a strength parameter given by

$$
y=\frac{2 Z_{t} Z_{c} m_{n} e^{2}}{\hbar v_{a}\left(m_{c}+m_{n}\right) b \kappa} .
$$

This formula shows very interesting scaling properties: very many experiments, for neutron halo nuclei with different binding energy, beam energy, and scattering angles (or $\boldsymbol{q}_{n}$ and $\boldsymbol{q}_{c}$ ), all lie on the same universal curve. The deviations from this simple scaling behavior, e.g., postacceleration effects, will lead to violations of such scaling.

\section{NUMERICAL RESULTS}

We now investigate the breakup of the one-neutron halo nuclei ${ }^{11} \mathrm{Be}$ and ${ }^{19} \mathrm{C}$. We take a heavy target of atomic number $Z=82$. In this paper, all the higher order results correspond to calculations performed within the finite-range CWBA model, while the first order results have been obtained by using Eq. (23). The structure term $Z_{l}^{\prime}$ [Eq. (21)] was calculated by adopting a single-particle potential model to obtain the ground state wave function of the projectile. The ground state of ${ }^{11} \mathrm{Be}$ was assumed to have a $2 s_{1 / 2}$ valence neutron coupled to the ${ }^{10} \mathrm{Be}\left(0^{+}\right)$core with a binding energy of $504 \mathrm{keV}$. The corresponding single particle wave function was constructed by assuming a neutron- ${ }^{10} \mathrm{Be}$ interaction of the Woods-Saxon type. For a set of values of the radius and diffuseness parameters (the same as those given in [8]), the depth of this potential was searched so as to reproduce the ground state binding energy. This $2 s_{1 / 2}$ wave function has an additional node as compared to a simpler zerorange wave function. For the ${ }^{19} \mathrm{C}$, the ground state was assumed to have a $\left[{ }^{18} \mathrm{C}\left(0^{+}\right) \otimes 2 s_{1 / 2} \nu\right]$ configuration with a separation energy of $0.530 \mathrm{MeV}$. The radius and diffuseness parameters, used in the well-depth search, were the same as those given in [8].

In Fig. 1, we present calculations for the triple-differential cross sections for the breakup reaction ${ }^{11} \mathrm{Be}+\mathrm{Pb} \rightarrow n+{ }^{10} \mathrm{Be}$ $+\mathrm{Pb}$, as a function of the energy of the ${ }^{10} \mathrm{Be}$ core $\left(E_{c}\right)$, for four beam energies lying in the range of 5-72 MeV/nucleon. To see the postacceleration in a clear way, it is very useful to study the cross section as a function of core energy. The results obtained within the higher order and first order theories are shown by solid and dotted lines, respectively.

It can be seen from this figure that while for lower beam energies the higher order and first-order results differ considerably from each other, they are almost the same for the beam energy of $72 \mathrm{MeV} /$ nucleon. In each case, the first order cross sections peak at the energy of the core fragment which corresponds to the beam velocity (this value of the core fragment energy will be referred to as $E_{b v}$ in the following). In contrast to this, the peaks of the higher order cross sections are shifted to energies $>E_{b v}$ for the three lower energies. Only for the $72 \mathrm{MeV} /$ nucleon beam energy does the higher order result peak at $E_{b v}$. This shows very clearly that the finite-range CWBA model exhibits postacceleration for beam energies $\leqslant 30 \mathrm{MeV} /$ nucleon, while this effect is not present 

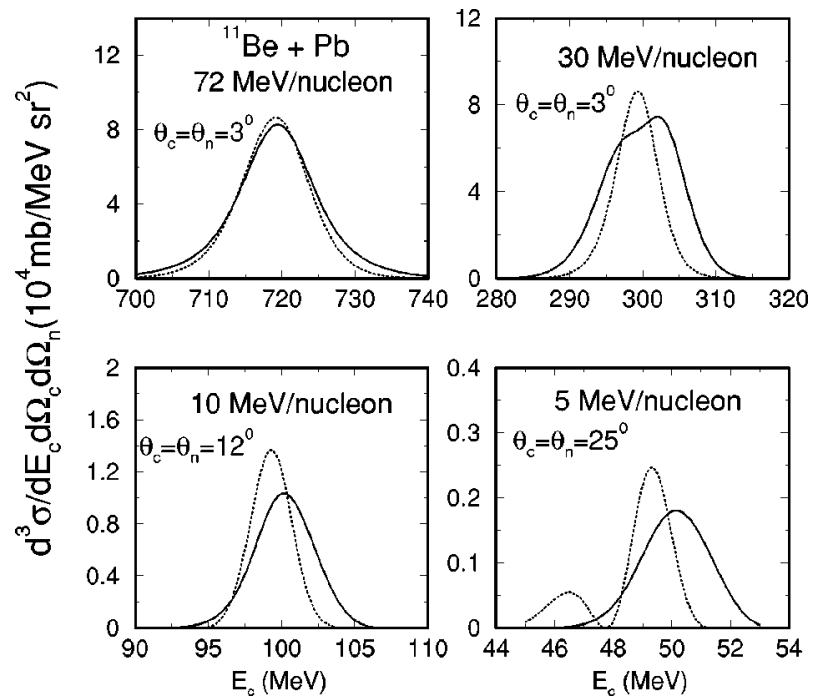

FIG. 1. Triple-differential cross section as a function of the energy of ${ }^{10} \mathrm{Be}$ core for the reaction ${ }^{11} \mathrm{Be}+\mathrm{Pb} \rightarrow n+{ }^{10} \mathrm{Be}+\mathrm{Pb}$ at beam energies of $72 \mathrm{MeV} /$ nucleon, $30 \mathrm{MeV} /$ nucleon, $10 \mathrm{MeV} /$ nucleon, and $5 \mathrm{MeV} /$ nucleon. The results of the finite-range CWBA and first order theory are shown by solid and dotted lines, respectively.

at $72 \mathrm{MeV} /$ nucleon. Therefore, the higher order effects are minimal for the Coulomb breakup of ${ }^{11} \mathrm{Be}$ at the beam energies $\geqslant 70 \mathrm{MeV}$. This result is in agreement with those obtained in $[19,20]$.

In Fig. 2, we compare the first order (dotted lines) and the higher order (solid lines) results for the double-differential cross section for the same reaction and for the same beam energies as in Fig. 1. These results have been obtained by integrating the triple-differential cross sections over the unobserved neutron solid angles. The cross sections are shown as a function of $E_{c}$. It is clear that for beam energies $\leqslant 30 \mathrm{MeV} /$ nucleon, the first order results peak at $E_{b v}$, but the higher order cross sections have their maxima at energies larger than $E_{b v}$. In contrast to this, both the higher order and first order cross sections peak at the same value of $E_{c}$ $\left(=E_{b v}\right)$, for the $72 \mathrm{MeV} /$ nucleon case. Therefore, the postacceleration effects are pronounced for the smaller beam energies, whereas they become quite small for the higher energies. The near equality of the first order and finite-range CWBA cross sections, at a beam energy of $72 \mathrm{MeV} /$ nucleon, suggest that for this reaction, the higher order effects, in general, are quite irrelevant at beam energies $\geqslant 70 \mathrm{MeV} /$ nucleon.

In Fig. 3, we compare the results of the first order and finite range CWBA calculations for the relative energy spectrum of the fragments emitted in the breakup reaction of

${ }^{11} \mathrm{Be}$ on a ${ }^{208} \mathrm{~Pb}$ target for the same four beam energies as shown in Fig. 2. These cross sections have been obtained by integrating over all the allowed values of the angles $\Omega_{c-n}$. In both the models, the integrations over $\theta_{t-(c+n)}$, have been carried out from $1^{\circ}$ to grazing angle, in the upper two figures, and from $5^{\circ}$ to grazing angles, in the lower two figures. The integrations over $\phi_{t-(c+n)}$ angles have been done over all of its kinematically allowed values. The dotted
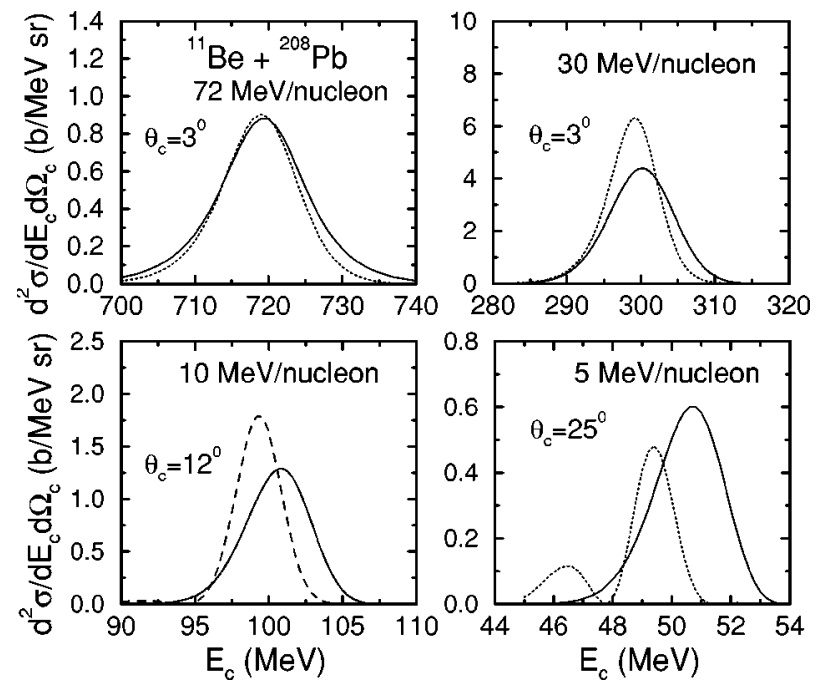

FIG. 2. Double-differential cross section as a function of the energy of ${ }^{10} \mathrm{Be}$ core for the reaction ${ }^{11} \mathrm{Be}+\mathrm{Pb} \rightarrow n+{ }^{10} \mathrm{Be}+\mathrm{Pb}$ at beam energies of $72 \mathrm{MeV} /$ nucleon, $30 \mathrm{MeV} /$ nucleon, $10 \mathrm{MeV} /$ nucleon, and $5 \mathrm{MeV} /$ nucleon. The results of the finite-range CWBA and first order theory are shown by solid and dotted lines, respectively.

and solid lines represent the results of the first order and higher order calculations, respectively. We notice that while for a beam energy of $72 \mathrm{MeV} /$ nucleon, the higher order effects are minimal, they are quite strong for the lower beam energies, being largest at the beam energy of $5 \mathrm{MeV} /$ nucleon. This reinforces the point, already made in $[19,20]$, that at a beam energy of $72 \mathrm{MeV} /$ nucleon, the higher order effects are quite small if both the first order and higher order terms are calculated within the same theory.

In Fig. 4, we show the same results as in Fig. 3, but for the ${ }^{19} \mathrm{C}$ induced reaction on the ${ }^{208} \mathrm{~Pb}$ target for the beam
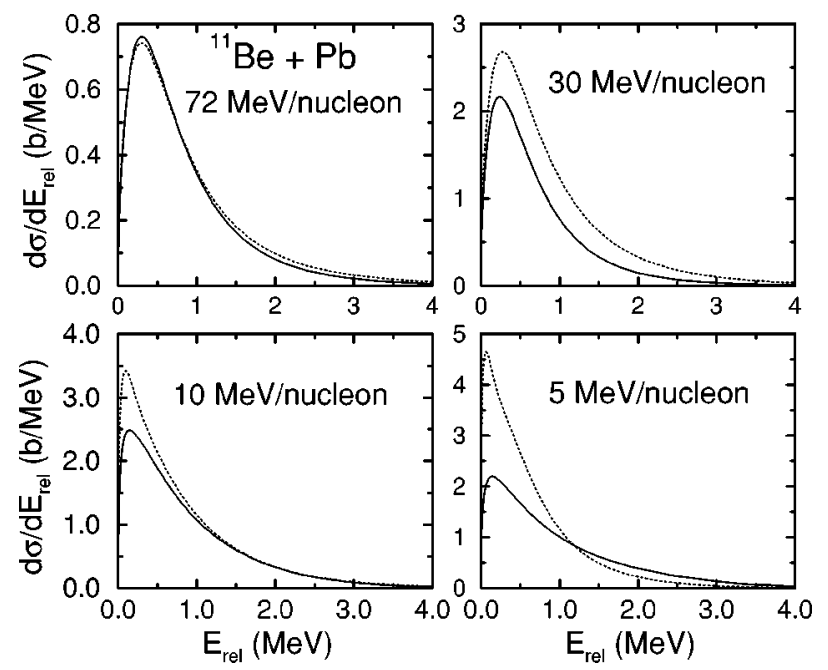

FIG. 3. The differential cross section as a function of the relative energy of the fragments (neutron and ${ }^{10} \mathrm{Be}$ ) emitted in the ${ }^{11} \mathrm{Be}$ induced breakup reaction on a ${ }^{208} \mathrm{~Pb}$ target at the beam energies of $72 \mathrm{MeV} /$ nucleon, $30 \mathrm{MeV} /$ nucleon, $10 \mathrm{MeV} /$ nucleon, and $5 \mathrm{MeV} /$ nucleon. The dotted and solid lines represent the first order and finite-range CWBA results, respectively. 


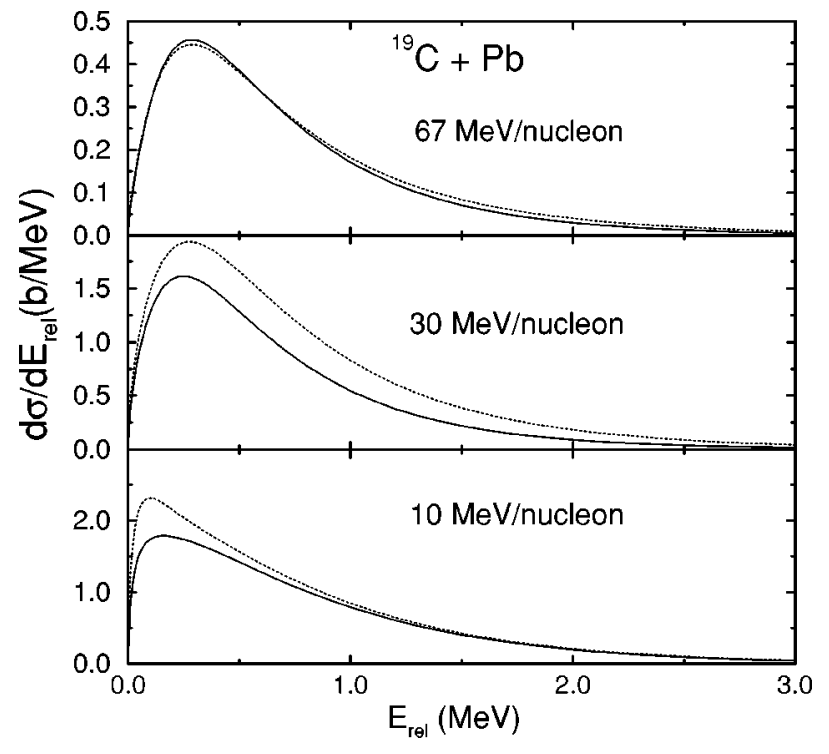

FIG. 4. The differential cross section as a function of the relative energy of the fragments (neutron and ${ }^{18} \mathrm{C}$ ) emitted in the ${ }^{19} \mathrm{C}$ induced breakup reaction on a ${ }^{208} \mathrm{~Pb}$ target at the beam energies of 67 $\mathrm{MeV} /$ nucleon, $30 \mathrm{MeV} /$ nucleon, and $10 \mathrm{MeV} /$ nucleon. The dotted and solid lines represent the first order and finite-range CWBA results, respectively.

energies of $67 \mathrm{MeV} /$ nucleon, $30 \mathrm{MeV} /$ nucleon, and $10 \mathrm{MeV} /$ nucleon. Like Fig. 3, the integrations over the fragments center of mass angles is done in the range from $1^{\circ}$ to grazing angle, for first two beam energies, and from $5^{\circ}$ to grazing angle, at the lowest beam energy. We see that in this case too the higher order effects are quite weak for a beam energy of $67 \mathrm{MeV} /$ nucleon, but appreciable for the lower beam energies.

It may be noted that by comparing the result of a conceptually different model of the Coulomb breakup reactions [36] than ours with that of the first order semiclassical perturbation theory of the Coulomb excitation, it has been concluded in [18] that the higher order effects are substantial for these reactions even at the beam energies of $\sim 70 \mathrm{MeV} /$ nucleon. However, one should be careful in drawing definite conclusions about the role of the higher order effects from such an approach. For a reliable assessment of the contributions of the higher order effects, it is essential that both the first order and higher order terms should be calculated within the same theory, as has been done in this work (and also in $[19,20]$ ).

\section{CONCLUSION AND OUTLOOK}

In this paper, we investigated the breakup of the oneneutron halo nuclei ${ }^{11} \mathrm{Be}$ and ${ }^{19} \mathrm{C}$ in the Coulomb field of a heavy target nucleus within a theory which is formulated in the framework of the post-form distorted wave Born ap- proximation. This theory contains the electromagnetic interaction between the core and target nucleus to all orders. An attractive feature of this formulation is that the corresponding pure Coulomb breakup amplitude can be expressed in an analytic form. We have also extracted the first order approximation of the full pure Coulomb breakup $T$ matrix. This enables us to investigate the respective roles of the first order and higher order effects within the same theoretical model. We studied the beam energy dependence of the first order and higher order triple- and double-differential cross sections. We also investigated the contributions of the higher order effects to the relative energy distribution of the fragments.

In the higher order model, the peaks in the triple- and double-differential cross sections versus core energy spectra are shifted to energies larger than those corresponding to the beam velocity at incident energies $\leqslant 30 \mathrm{MeV} /$ nucleon. Therefore, postacceleration effects are important at these beam energies. On the other hand, at the beam energy $\sim 70 \mathrm{MeV} /$ nucleon, the corresponding spectra peak at the beam velocity energies, which is consistent with no postacceleration. In contrast to this, the first order cross sections always peak at the beam velocity energy, which is expected as the postacceleration is a higher order effect.

The higher order effects are also found to be quite important in the relative energy spectrum of the fragments at beam energies $\leqslant 30 \mathrm{MeV} /$ nucleon, while they are insignificant at the beam energies $\sim 70 \mathrm{MeV} /$ nucleon. This suggests that the conclusions arrived at in Refs. [12,13], where the data on the relative energy spectra of the fragments taken in the breakup of ${ }^{11} \mathrm{Be}$ and ${ }^{19} \mathrm{C}$ at beam energies $\sim 70 \mathrm{MeV}$ have been analyzed within the first order theory of the Coulomb excitation, may not be affected by the higher order effects.

The present model can be seen as a "theoretical laboratory," which allows us to study numerically the relation between quantal and semiclassical theories, and the importance of postacceleration effects. It should be noted that from an experimental point of view, the postacceleration effects are not fully clarified (see, e.g., $[12,37,38])$. Finally, let us mention the recent work on the electromagnetic dissociation of unstable neutron-rich oxygen isotopes [39]. These authors deduce photoneutron cross sections from their dissociation measurements. If the neutrons are emitted in a slow evaporation process in a later stage of the reaction, the question of postacceleration is not there. On the other hand, for the light nuclei there is some direct neutron emission component and the present kind of theoretical analysis further proves the validity of the semiclassical approach used in [39].

Postacceleration effects are also of importance for the use of Coulomb dissociation for the study of radiative capture reactions of astrophysical interest. We expect that our present investigations will shed light on questions of postacceleration and higher order effects in these cases also.
[1] P.G. Hansen, A.S. Jensen, and B. Jonson, Annu. Rev. Nucl. Part. Sci. 45, 2 (1995).

[2] I. Tanihata, J. Phys. G 22, 157 (1996), and references therein.

[3] G. Baur, S. Typel, and H. Wolter, in Spins and Nuclear and
Hadronic Reaction, Proceedings of the RCNP-TAMU Symposium, edited by H. Yabu, T. Suzuki, and H. Toki (World Scientific, Singapore, 2000), p. 119.

[4] R. Shyam and P. Danielewicz, Phys. Rev. C 63, 054608 
(2001).

[5] C.A. Bertulani and G. Baur, Nucl. Phys. A480, 615 (1988); M.S. Hussein, M.P. Pato, and C.A. Bertulani, Phys. Rev. C 44, 2219 (1991).

[6] G.F. Bertsch and H. Esbensen, Ann. Phys. (N.Y.) 209, 327 (1991).

[7] G. Baur and H. Rebel, Annu. Rev. Nucl. Part. Sci. 46, 321 (1996), and references therein.

[8] R. Chaterjee, P. Banerjee, and R. Shyam, Nucl. Phys. A675, 477 (2000).

[9] C.A. Bertulani, G. Baur, and M.S. Hussein, Nucl. Phys. A526, 751 (1991)

[10] G. Baur, F. Rösel, D. Trautmann, and R. Shyam, Phys. Rep. 111, 333 (1984).

[11] K. Alder and A. Winter, Electromagnetic Excitation (NorthHolland, Amsterdam, 1975).

[12] T. Nakamura et al., Phys. Lett. B 331, 296 (1994).

[13] T. Nakamura et al., Phys. Rev. Lett. 83, 1112 (1999).

[14] G.F. Bertsch and C.A. Bertulani, Nucl. Phys. A556, 136 (1993); C.A. Bertulani and G.F. Bertsch, Phys. Rev. C 49, 2839 (1994).

[15] H. Esbensen, G.F. Bertsch, and C. A. Bertulani, Nucl. Phys. A581, 107 (1995).

[16] T. Kido, K. Yabana, and Y. Suzuki, Phys. Rev. C 53, 2296 (1996).

[17] V.S. Melezhik and D. Baye, Phys. Rev. C 59, 3232 (1999).

[18] J.A. Tostevin, in Proceedings of the Second International Conference on Fission and Properties of Neutron-rich Nuclei, St. Andrews, Scotland, 1999, edited by J.H. Hamilton, W.R. Phillips, and H.K. Carter (World Scientific, Singapore, 2000), p. 429.
[19] S. Typel and G. Baur, Phys. Rev. C 64, 024601 (2001).

[20] S. Typel and R. Shyam, Phys. Rev. C 64, 024605 (2001).

[21] G. Baur and D. Trautmann, Nucl. Phys. A191, 321 (1972).

[22] R. Shyam, P. Banerjee, and G. Baur, Nucl. Phys. A540, 341 (1992).

[23] N. Austern, Direct Reaction Theory (Wiley, New York, 1970).

[24] N. Glendenning, Direct Nuclear Reaction (Academic, New York, 1983).

[25] G.R. Satchler, Nucl. Phys. 5, 1 (1964).

[26] R. Shyam and M.A. Nagarajan, Ann. Phys. (San Diego) 163, 285 (1985).

[27] P. Braun-Munzinger and H.L. Harney, Nucl. Phys. A233, 381 (1974).

[28] R. Chatterjee, P. Banerjee, and R. Shyam, Nucl. Phys. A692, 476 (2001)

[29] A. Nordsieck, Phys. Rev. 93, 785 (1954).

[30] F. Fuchs, Nucl. Instrum. Methods Phys. Res. 200, 361 (1982).

[31] G. Baur, C.A. Bertulani, and D.M. Kalassa, Nucl. Phys. A550, 107 (1995).

[32] G. Baur and D. Trautmann, Phys. Rep., Phys. Lett. 25C, 293 (1976).

[33] P. Banerjee and R. Shyam, Nucl. Phys. A561, 112 (1993).

[34] G. Baur, K. Hencken, D. Trautmann, S. Typel, and H.H. Wolter, Prog. Part. Nucl. Phys. 46, 99 (2001).

[35] G. Baur, K. Hencken, and D. Trautmann, nucl-th/0108013.

[36] J.A. Tostevin, S. Rugmai, and R.C. Johnson, Phys. Rev. C 57, 3225 (1998); J.A. Tostevin et al., Phys. Lett. B 424, 219 (1998).

[37] K. Ieki et al., Phys. Rev. Lett. 70, 730 (1993).

[38] J.E. Bush et al., Phys. Rev. Lett. 81, 61 (1998).

[39] A. Leistenschneider et al., Phys. Rev. Lett. 86, 5442 (2001). 